\title{
Effect of Skin-To-Skin Contact on Preterm Infant Skin Barrier Function and Hospital-Acquired Infection
}

\author{
Amel Abouelfettoh ${ }^{\mathrm{a}, \mathrm{b}, \mathrm{d}}$, Susan M. Ludington-Hoe ${ }^{\mathrm{a}}$, Chris J. Burant ${ }^{\mathrm{a}}$, Marty O. Visscher ${ }^{\mathrm{c}}$
}

\begin{abstract}
Background: The preterm infants' skin is structurally and functionally immature at birth because of immature stratum corneum barrier function, leading to problems with fluid loses, thermoregulation, and infection. Two parameters of barrier function can be non-invasively assessed: Stratum Corneum Hydration ( $\mathrm{SCH})$ and Transepidermal Water Loss (TEWL). Skin-to-Skin Care (SSC) is the proposed independent variable that might affect barrier function by decreasing TEWL and increasing $\mathrm{SCH}$, thereby improving stratum corneum barrier function and consequently decreasing the rate of infection. No study of SSC's effects on TEWL and SCH of preterm infants could be found. The purpose of the study was to determine the effect of 5 daily Skin-to-Skin Contact sessions on infant skin hydration $(\mathrm{SCH})$, transepidermal evaporated water loss (TEWL), and on SCH when TEWL was controlled, and on the presence of hospital acquired infection.
\end{abstract}

Methods: A one-group pretest-test-posttest design with 10 preterm infants $(28-30$ wks GA $<32$ wks postmenstrual age, and no infection at entry). Test $=90$ minutes of SSC; pre-test and post-test $=30$ minutes each of prone positioning in an incubator. SCH and TEWL were taken on Days 1 and 5 at the beginning, middle and end of each period using Multi-Probe Adaptor. A 3 X 3 X 2 Repeated Measures Mixed Models Design, including a covariate, was used to analyze level of Skin Hydration. Specifically, the model tested comparisons in $\mathrm{SCH}$ made across repetitions, time, and days,

Manuscript accepted for publication December 16, 2010

${ }^{\text {a }}$ Case Western Reserve University Bolton School of Nursing, USA

${ }^{\mathrm{b}}$ Cairo University Faculty of Nursing, Egypt

'Skin Sciences Institute, Cincinnati Children's Hospital Research Foundation, USA

${ }^{\mathrm{d} C}$ Corresponding author: Amel Abouelfettoh, College of Nursing, King Saud Bin Abdulaziz University for Health Sciences, National Guard Health Affairs (NGHA), Mail Code: 500, P.O. Box 2477, Al-Ahsa-31982 KSA. Email: amelmoataz@hotmail.com

doi:10.4021/jocmr479w as well as all possible interactions while controlling for TEWL. Descriptive statistics described the number of positive blood cultures during hospitalization and the presence of infections four weeks post-discharge.

Results: Significant differences in skin hydration were found across TIME (Pre-SSC, SSC, Post-SSC) $(\mathrm{F}=21.86 ; \mathrm{p}<0.001)$. One infant had a positive blood culture during hospitalization; no infants had signs of infection by 4 weeks post-discharge.

Conclusions: The study has begun fulfilling the recommendation that SSC be tested as a strategy to improve skin hydration, but reveals that evaporative loss may be higher during SSC than during incubator care, and that the higher transepidermal evaporated water loss values may not necessarily be detrimental because few infections occurred even in its presence. A definitive randomized controlled trial is recommended.

Keywords: Skin-to-skin contact; Skin hydration; Transepidermal water loss; Infection; Preterm

\section{Introduction}

Because the skin is a common point of entry for invasive pathogens, interventions to improve the preterm infant's skin barrier functions are needed [1]. The skin of premature infants has the functions of inhibiting water loss (called transepidermal evaporated water loss (TEWL)) from the tissues, and maintaining adequate moisture (stratum corneum hydration $(\mathrm{SCH})$ ) through the stratum corneum (SC) to prevent entry of damaging microorganisms. But, skin barrier functions are compromised due to immaturity [2]. The premature infant's immature skin lacks vernix casseosa, a protective cutaneous biofilm with antimicrobial properties [3]. When prematurity becomes more extreme, the skin barrier is increasingly immature, the skin is more easily damaged and more functionally compromised [4]. The SC layer is also thinner and less well-developed than in full-term infants and adults, leading to high water loss, cutaneous infections, and high morbidity and mortality [5]. Consequently, hospitalized preterm infants suffer from the risks of infection and infec- 
tion's co-morbidities [6-10]. Infection per se in preterm infants is also associated with increased risk of death [1] and increased medical cost [11-13].

Successful interventions to reduce infections, especially hospital acquired infections, would improve survival, reduce neonatal morbidity, and reduce the high medical and social costs of preterm infant care [5, 14-16]. Many interventions to reduce infection have been tested and are in use, such as hand-washing, administration of antibiotics and immunoglobulins, and isolation of the infant $[2,15,17-20]$, but infections continue to occur. However, preterm infants experiencing skin-to-skin contact (SSC) have had fewer infections than infants who did not receive SSC $[21,22]$.

Skin-to-skin contact is skin-to-skin, chest-to-chest placement of the infant with mother at an incline of $30-40$ degrees. Skin-to-skin contact may help reduce infections by improving the preterm infant's skin barrier function. A decrease in TEWL and an increase in SCH along all areas of infant skin that are in direct contact with maternal skin could occur and would be indicators of improved skin barrier function. Intermittent exposure to a high-humidity environment during feeding has been shown to quicken maturation of the skin barrier [23], leading the authors to hypothesize that intermittent exposure to the high-humidity microenvironment of SSC may do the same. No study of SSC's effects on TEWL and SCH of preterm infants could be found. Thus, the purposes of the study were to test for differences in TEWL and SCH between incubator (control condition) and SSC (experimental condition) on the first and last day of five daily SSC sessions within the same subjects, with each subject acting as his own control, and to determine the effect on SCH when TEWL was statistically controlled because $\mathrm{SCH}$ is a function of TEWL. The hypotheses were that 1) TEWL would be lower and $\mathrm{SCH}$ would be higher in SSC than in the incubator on Days 1 and 5, and 2) differences on Day 5 would be more favorable (lower TEWL and higher $\mathrm{SCH}$ ) than differences on Day 1. One research question was posed: (because improved SCH and TEWL would enhance skin barrier function) what was the number of hospital-acquired infections (number of positive blood cultures during hospitalization and maternal report of any signs of infection at four weeks post-discharge) in infants who received SSC?

\section{Theoretical framework (relationship between SSC and skin barrier function)}

Skin-to-skin contact may enhance SC barrier function in two ways. The first way is that TEWL may be minimized due to decreased skin exposure to evaporative heat loss during SSC [24, 25] or due to the "heat sink" microenvironment encountered when the infant lies contained between maternal breasts [26]. The environment between maternal breasts is a "heat sink" because the humidity and temperature are higher between the breasts than they are in an incubator, thereby preventing evaporative losses across the skin [27]. The second way in which SC barrier function may be enhanced is that the higher humidity microenvironment of SSC may reduce TEWL [28], resulting in a subsequent increase in skin hydration, a finding suggested by reports that mothers have commented about "sweating" during SSC, so that SSC is expected to provide the infant with more hydration resulting from mother's sweats [26, 29].

\section{Methods}

\section{Design}

A one-group, preSS-SSC-postSSC quasi-experimental one group design was conducted in which each subject served as his/her own control. Premature infants were tested during one inter-feeding interval on Days 1 and 5 of a five-day study in which infants were given SSC each day for five consecutive days. The inter-feeding interval was divided into three periods: a 30-minute pre-SSC period in the incubator, followed by a 90 -minute SSC period, and then another 30 -minute post-SSC period in the incubator immediately after SSC ended. On Days 1 and 5, TEWL and SCH were assessed three times in each period: at 10, 20, and 30 minutes of the pre-SSC and post-SSC periods, and at 30,60, and 90 minutes during the SSC period, so that early, middle, and end assessment data for each period were available. On Days 2, 3, and 4, SSC was given for at least 30 and no more than 120 minutes each day. Signed informed consent was obtained following institutional review board approval at a university hospital in the Midwest. One research associate administered all assessments and monitored computerized data acquisition.

\section{Setting}

Preterm infants were tested in a tertiary university-based Neonatal Intensive Care Unit which consisted of 7 rooms, each room housing 3 - 5 preterm infants of varying severity of illness who were separated by screens. Ambient temperature and humidity were recorded immediately before each TEWL and SCH reading during the pre-SSC, SSC, and postSSC periods.

\section{Sample}

Twenty-five mother-infant pairs were eligible over a 12 months period and 17 gave consent. Seven of the consented pairs did not begin data collection because of infant illness $(\mathrm{n}=3)$ or withdrawal at maternal request due to hardship in providing 5 consecutive days of SSC $(n=4)$. Convenience sampling resulted in 10 mother-infant pairs who provided complete data sets. 


\section{Subjects}

Preterm infants born between 28 - 30 weeks gestation who met the following criteria were recruited: 1) were $30-31$ weeks postmenstrual age at time of entry, so one week of study could be completed prior to the infant being 33 weeks post-conception age, the age at which the stratum corneum is usually as mature as the term infant's skin [23, 30], 2) were no more than 2 and 2/7 weeks old because the stratum corneum matures by completion of the third week of life [4] in infants living in an environment with a relative humidity less than $85 \%$ [23] which was the condition for all infants in the study, 3) were not receiving oxygen support, so transfer into and out of SSC was not complicated by oxygen lines, and 4) were not having central lines at time of study because central lines could be a source of infection as suggested by Asembergiene and colleagues [31]. Mothers were at least 18 years of age so they could give consent, were able to speak English so they could give informed consent, were willing and able to come for 5 consecutive days for SSC, and had no signs of skin rash or upper respiratory infection at time of entry. Infant exclusion criteria were 1) a previous infection any time since birth because previous infection indicates high susceptibility to re-infection [31], and 2) an impending (as determined by an increase in apnea, bradycardia and desaturation episodes, poor feeding, irritability, or lethargy) or active infectious process (as determined by positive blood cultures and/or administration of antibiotics and/or a diagnosis to rule out infection).

\section{Conditions}

Feedings for all subjects were scheduled every three hours. For preSSC and postSSC periods the infants remained in double-walled incubators, and were positioned flat, prone, and nested while wearing only a diaper and a head cap. Transfer into SSC was accomplished by sitting transfer and infants were placed skin-to-skin, chest-to-chest between the breasts so that the infant's head was above the level of the mother's nipples and the head was turned to one side and erect (not flexed). During SSC infants wore diapers and had a receiving blanket folded-in-fourth covering their backs. The mother's hospital gown was closed over the infant's back. Mothers reclined at a comfortable level in a stationary zero-gravity lounger (La Fuma, France) behind privacy screens. Between Days 1 and 5 mothers agreed to provide daily sessions of SSC that were at least 65 minutes long.

\section{Outcome measures}

$\mathrm{SCH}$ was defined as the amount of water retained by stratum corneum cells in the skin. Stratum corneum hydration is measured by changes in skin surface electrical capacitance and determined by the Moisture Accumulation Test [32].
$\mathrm{SCH}$ was measured as the moisture accumulation using the Corneometer ${ }^{\circ}$ CM 825 [33]. The Corneometer probe was placed vertically against the infant's skin without pressure at a mark two centimeters below the infant's left nipple at the midclavicular line. The probe was held in constant contact for six seconds; $\mathrm{SCH}$ was recorded every second. The Corneometer-probe started the $\mathrm{SCH}$ measurement as soon as it came in contact with skin. A beep signaled that the $\mathrm{SCH}$ measurement had been successfully obtained, and once six measurements were obtained the probe was removed.

TEWL was defined as the rate of water evaporation from the skin and reported as $\mathrm{g} / \mathrm{m}^{2} / \mathrm{hr}$. Because TEWL is reported as a rate of evaporation rather than an amount of evaporation, TEWL was computed by averaging the data recorded every one second for 20 - 120 seconds determined by the shortest time to achieve 5 consecutive readings with a standard deviation $<0.1$. The TEWL was measured by a Tewameter ${ }^{\circledR}$ TM 300 [33]. The TEWL probe was warmed up for 2 minutes before the first TEWL measurement was taken, and then the probe was turned on and manually held constantly against the infant's skin without pressure two centimeters below the infant's left nipple at the midclavicular line. The probe was maintained on the infant's skin for at least 20 seconds and no more than 120 seconds, detecting one measurement every second until the standard deviation between 5 consecutive readings was $<0.1$, at which time the device automatically terminated readings [33].

Number of infections was defined as number of positive blood cultures since the day of entry into the study to the day of infant discharge from the hospital. Positive blood cultures were determined by examining daily lab reports.

Signs and symptoms of infection after discharge were defined as a positive maternal response to any one or more of the following questions: Has your infant had any fever since leaving the neonatal intensive care unit? Has your infant been diagnosed as having an infection since leaving the neonatal intensive care unit? Has your infant received any antibiotics during the first thirty days after leaving the neonatal intensive care unit?

\section{Instruments}

To measure TEWL and SCH, a Multi-probe Adapter Systems MPA ${ }^{\circledR}$ [33] were used. The MPA system accommodated two probes: one probe was the Corneometer ${ }^{\circledR}$ CM 825 probe to measure $\mathrm{SCH}$. The other probe was the Tewameter ${ }^{\circledR}$ TM 300 probe to measure TEWL. The MPA system was connected to a laptop computer with MPA software. SCH and TEWL measures were continuously downloaded from the MPA to the computer in real time in Excel format. The Tewameter and Corneometer probes did not need recalibration with each use, but calibration of the probes was checked once every month. The accuracy of the Tewameter probe was $\pm 0.5 \mathrm{~g} / \mathrm{hm}^{2}$ for Relative Humidity $\geq 30 \%$ and $\pm 1.0 \mathrm{~g} /$ 
$\mathrm{hm}^{2}$ for Relative Humidity $\leq 30 \%$. The accuracy of the Corneometer probe was $\pm 3.0 \%$ at optimal Relative Humidity of $30 \%-70 \%$. To ensure accurate function of the MPA probes, the MPA system was stored under the recommended storage conditions (Temperature: $0-70{ }^{\circ} \mathrm{C}$, Relative Humidity: $0 \%-80 \%$ ). Probes were warmed up for two minutes before measurements were taken and were not removed from the neonatal intensive care unit once the study began.

A Fisher Scientific Traceable ${ }^{\circledR}$ Thermistor/Clock/Humidity Monitor (Model 06-662-4) was used to measure ambient temperature and relative ambient humidity. The monitor's accuracy was $\pm 1{ }^{\circ} \mathrm{C}$ for ambient temperature, and \pm $4 \%$ for relative humidity, and was calibrated biennially by the manufacturer.

Ohmeda Care Plus incubator skin probe was used to obtain infant skin temperature. The probe was placed one centimeter below the right costal margin and covered by a Mylar temperature shield (Accutemp Plus, Hayward, CA) to minimize ambient air and light influences. The probe is a 1.5 centimeter diameter flat metal disk that was attached to the abdomen one centimeter below the right costal margin at the mid-clavicle line.

\section{Procedure}

Following signed consent, mothers and the research associate mutually agreed to a start date for SSC (Day 1). All measurements were taken on Days 1 and 5. The infant was positioned prone and left undisturbed in the incubator for the pre-SSC period. Ambient temperature and relative humidity were recorded at the beginning of each period. Assessments of infant skin temperature, TEWL, and $\mathrm{SCH}$ were recorded at Minutes 10, 20, and 30 of the pre-SSC and post-SSC periods. The SSC period began as soon as infant was transferred to mother's chest. Infant abdominal skin temperature, $\mathrm{SCH}$, and TEWL assessments were recorded at Minutes 30, 60, and 90 of the SSC period. At the end of SSC, the infant was transferred back to the incubator and positioned the same as in the pre-SSC period. Infants were undisturbed by staff between measurements. On Days 2, 3, and 4, infants received SSC for 65 - 120 minutes, beginning whenever the mother came to visit. SSC was given only once each day.

\section{Data analysis}

Subjects' demographics, TEWL, SCH, number of positive blood cultures during hospitalization, and the presence of signs of infection four weeks post-discharge were described using measures of central tendency and dispersion (Mean and SD). In the current study, data were collected from 10 subjects over 18 time points. The main focus was comparing Skin Hydration across three TIME periods (Pre-SSC, SSC, and Post-SSC). In order to take advantage of collecting data at 18 time points, a Repeated Measures Mixed Model ap- proach was used to analyze the data, an approach that provided more power to analyze the data because the effective sample became 180 data points ( 10 subjects X 18 data collection points) as compared to 10 data points based on subjects alone. Data were collected in three ASSESSMENTS of data collection points for each of the three TIME periods across two DAYS. With this in mind, the final model was based on a 3 X 3 X 2 Repeated Measures Mixed Model design. This model allowed for the testing of the three ASSESSMENTS of data collection points across three PERIODS across two DAYS. Typically, in using a Repeated Measures Mixed Models Approach, an error covariance structure has to be identified for the repeated time components of the model. In the Current Model, a Compound Symmetry error covariance structure was applied to the PERIOD component. Testing of the ASSESSMENTS component indicated that an error covariance structure did not need to be applied as ASSESSMENTS demonstrated an independent error structure. Additionally, TEWL was included as a covariate, because TEWL impacts levels of Stratum Corneum Hydration. With this model, comparisons in SCH can be made across ASSESSMENTS, PERIODS, and DAYS, as well as the testing of interactions comparing ASSESSMENTS across PERIODS, ASSESSMENTS across DAYS, PERIODS across DAYS, and ASSESSMENTS across PERIODS across DAYS.

Table 1. Mothers and Infants Demographic Characteristics $(\mathrm{N}=10)$

\begin{tabular}{lll}
\hline Characteristics & Mean \pm SD & N \\
\hline Mothers Age & $24.6 \pm 4.03$ & \\
Infants GA & $28.6 \pm 1.26$ & \\
Infants Age at Entry & $15.2 \pm 7.19$ & \\
Minutes of KC/day (Day 2 - 4) & $77.5 \pm 12.5$ & \\
Infants Birth Weight & $1228.4 \pm 167.6$ & \\
Infants Weight at day one & $1183.7 \pm 331.8$ & \\
Infants Weight at day five & $1348 \pm 173.9$ & \\
Male/Female & & $9 / 2$ \\
AA/Caucasian & & \\
Vaginal/Cesarean Delivery & & \\
Breastfeeding/No Breastfeeding & & \\
\hline
\end{tabular}


Table 2. Means \pm SDs for Ambient Humidity and Temperature and Infant Skin Temperature During Study Periods on Days 1 and 5

\begin{tabular}{|c|c|c|c|c|c|c|}
\hline & \multicolumn{3}{|c|}{ Day 1} & \multicolumn{3}{|c|}{ Day 5} \\
\hline & Pre-SSC & SSC & Post-SSC & Pre-SSC & SSC & Post-SSC \\
\hline $\begin{array}{l}\text { Ambient } \\
\text { Humidity }\end{array}$ & $44.9 \pm 12.2$ & $27.3 \pm 7.1$ & $38.6 \pm 12.7$ & $39.1 \pm 11.7$ & $28.8 \pm 7.3$ & $36.1 \pm 9.0$ \\
\hline $\begin{array}{l}\text { Ambient } \\
\text { Temperature }\end{array}$ & $30.7 \pm 0.7$ & $27.2 \pm 0.7$ & $30.3 \pm 0.9$ & $30.2 \pm 1.9$ & $27.1 \pm 1.3$ & $29.9 \pm 2.1$ \\
\hline $\begin{array}{l}\text { Infant Skin } \\
\text { Temperature }\end{array}$ & $36.7 \pm 0.3$ & $36.8 \pm 0.2$ & $36.7 \pm 0.3$ & $36.7 \pm 0.4$ & $37.0 \pm 0.2$ & $36.6 \pm 0.2$ \\
\hline
\end{tabular}

${ }^{*} p<0.001$ across all periods during both days

TEWL was also analyzed using the same 3 X 3 X 2 Repeated Measures Mixed Model design. As with the previous model, a Compound Symmetry error covariance structure was applied to the TIME component, while the ASSESSMENTS component had an independent error structure.

\section{Results}

Infant and maternal characteristics are presented in Table 1. Of note was the racial distribution of the subjects because 9 of the 10 subjects were Black. The total amount of SSC received daily on Days 2 - 4 was a mean of $77.5(\mathrm{SD}=12.5)$ minutes. Ambient temperatures and infant skin temperature were not significantly different across all periods on Days 1 and 5. However, ambient humidity, as expected, was higher in the incubators than in the rooms during SSC $(\mathrm{p}=0.001)$ (Table 2).

\section{$\mathrm{SCH}$}

$\mathrm{SCH}$ mean values during each period on Days 1 and 5 are presented in Table 3. SCH demonstrated a pattern of increase from pre-SSC to SSC and then a decrease from SSC to postSSC on Days 1 and 5 (Fig. 1, 2). A 3 X 3 X 2 Repeated Measures Mixed Models Design including a covariate was used to analyze level of SCH. Specifically, the model tested comparisons in SCH made across ASSESSMENTS, PERIODS, and DAYS, as well as all possible interactions while controlling for TEWL. In examining the main effects differences in ASSESSMENTS in data collection points and DAYS of SCH were not significant. However, significant differences in SCH were found across PERIODS (Pre-SSC, SSC, Post-SSC $)(F(2,18)=21.86 ; p<0.001)$. It should be noted that all mean scores are based on Least Squares Mean estimates. In examining the differences in SCH across PERIODS, SCH scores during the Pre-SSC period (LSMeans $=37.41$ ) were significantly lower than $\mathrm{SCH}$ scores during the SSC period (LSMeans $=53.87)($ Tukey-Kramer adjusted $\mathrm{p}<0.001)$. Stratum corneum hydration scores during the Pre-SSC period $($ LSMeans $=37.41)$ were significantly lower than $\mathrm{SCH}$ scores during the Post-SSC period (LSMeans = 48.68) (Tukey-Kramer adjusted $\mathrm{p}<0.001$ ). However, only a trend was found comparing SCH scores during the SSC period $(\mathrm{LSMeans}=53.37)$ and $\mathrm{SCH}$ scores during the PostSSC period $($ LSMeans $=48.68)($ Tukey-Kramer adjusted $\mathrm{p}=$ 0.09). All of these scores were adjusted for TEWL, however a non-significant trend was found for TEWL's influence on $\operatorname{SCH}(\mathrm{F}(1,152)=3.72 ; \mathrm{p}=0.06)$.

With regard to interaction effects, the only significant interaction effect was based on comparisons in SCH across ASSESSMENTS in data collection points and PERIODS (Pre-SSC, SSC, Post-SSC) $(\mathrm{F}(4,36)=2.83 ; \mathrm{p}<0.05)$. Among the 36 comparisons based on interactions, 10 significant differences were identified. Consistently, $\mathrm{SCH}$ at

Table 3. SCH Means and SD Across Periods for Day 1 and Day 5 of the Study

\begin{tabular}{llll}
\hline & $\begin{array}{l}\text { Pre-SSC } \\
\text { Mean } \pm \text { SD }\end{array}$ & $\begin{array}{l}\text { SSC } \\
\text { Mean } \pm \text { SD }\end{array}$ & $\begin{array}{l}\text { Post-SSC } \\
\text { Mean } \pm \text { SD }\end{array}$ \\
\hline Day one & $35.53 \pm 14.69$ & $53.06 \pm 15.10$ & $46.85 \pm 12.67$ \\
Day five & $37.02 \pm 7.56$ & $56.24 \pm 17.84$ & $51.22 \pm 13.86$ \\
\hline
\end{tabular}




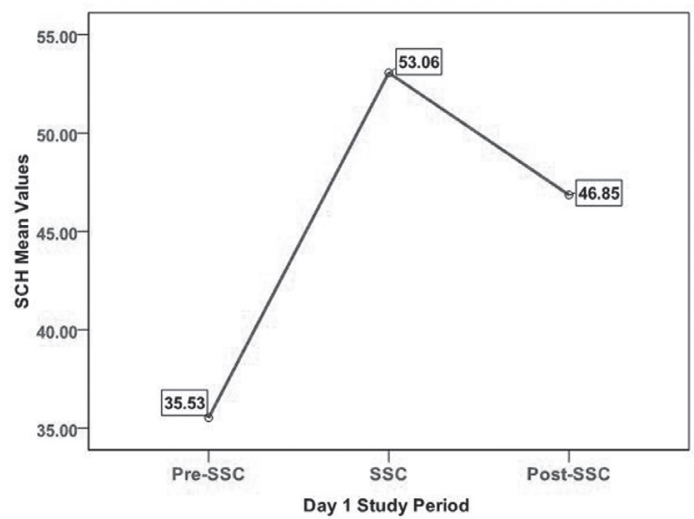

Figure 1. SCH on Day 1 across study periods.

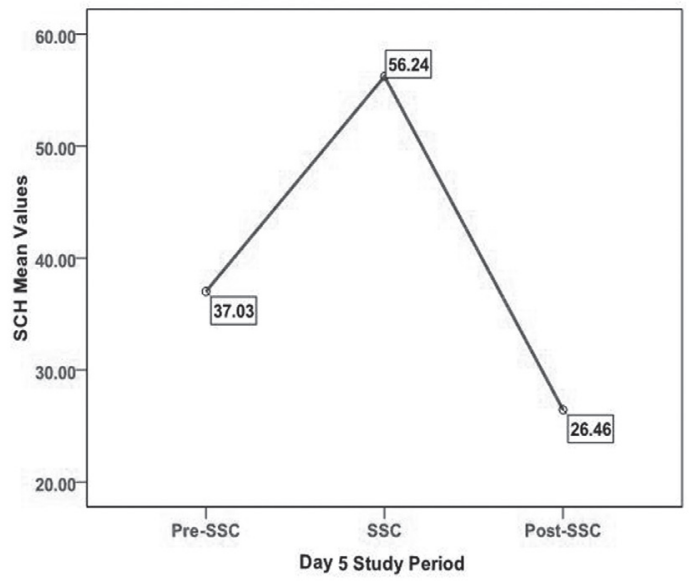

Figure 2. SCH on Day 5 across all study periods. the second and third assessments during SSC and at the first assessment of Post-SSC were significantly larger as compared across assessments during Pre-SSC (Table 4). The first $\mathrm{SCH}$ assessment during SSC was not significantly different from any of the three assessments in Pre-SSC. As previously mentioned, the second SCH assessment during SSC was significantly larger than the first (Tukey-Kramer adjusted $\mathrm{p}<$ 0.05 ), the second (Tukey-Kramer adjusted $\mathrm{p}<0.01$ ), and the third (Tukey-Kramer adjusted $\mathrm{p}<0.01$ ) assessments during Pre-SSC. The third SCH assessment during SSC was significantly larger than the first (Tukey-Kramer adjusted $\mathrm{p}<$ 0.01 ), the second (Tukey-Kramer adjusted $\mathrm{p}<0.001$ ), and the third (Tukey-Kramer adjusted $\mathrm{p}<0.001$ ) assessments during Pre-SSC. A similar trend in comparing the first $\mathrm{SCH}$ assessment during Post-SSC and the three Pre-SSC assessments was found. Specifically, the first Post-SSC assessment was significantly larger than the first (Tukey-Kramer adjusted $\mathrm{p}<0.05$ ), the second (Tukey-Kramer adjusted $\mathrm{p}<0.01$ ), and the third (Tukey-Kramer adjusted $\mathrm{p}<0.01$ ) assessments during Pre-SSC. Additionally, the third SCH assessment during SSC was significantly larger than the third assessment of Post-SSC (Tukey-Kramer adjusted $\mathrm{p}<0.05$ ). These findings reflect that $\mathrm{SSC}$, as compared to Pre-SSC, increased levels of SCH not significantly at the first assessment, but significantly through the second and third assessments during SSC and carried over onto the first assessment of Post-SSC. Interestingly, this carry over did not continue onto the third assessment of Post-SSC, where SCH levels were significantly lower as compared to the third assessment during SSC.

Table 4. Least Squares Means of SCH - Pair Wise Comparisons of PreSSC Period by SSC Period and PostSSC Period (Based on the Interaction Between Assessments and Periods)

\section{Pre-SSC Assessment 1 Pre-SSC Assessment 2 Pre-SSC Assessment 3}

\begin{tabular}{lllll}
\hline & & LS Mean $=38.42$ & LS Mean $=36.55$ & LS Mean $=37.26$ \\
$\begin{array}{l}\text { SSC } \\
\text { Assessment 1 }\end{array}$ & LS Mean $=49.56$ & Ns & Ns & Ns \\
SSC & LS Mean $=54.75$ & $P<0.05$ & $P<0.01$ & $P<0.01$ \\
$\begin{array}{l}\text { Assessment 2 } \\
\text { SSC }\end{array}$ & LS Mean $=57.30$ & $P<0.01$ & $P<0.001$ & $P<0.001$ \\
Assessment 3 & LS Mean $=53.82$ & $P<0.05$ & $P<0.01$ & $P<0.01$ \\
Post-SSC Assessment 1 & LS Mean $=49.52$ & Ns & Ns & Ns \\
Post-SSC Assessment 2 & LS & Ns & Ns \\
Post-SSC Assessment 3 & LS Mean $=42.72$ & Ns & N
\end{tabular}


Table 5. TEWL Means and SDs Across Periods for Day 1 and Day 5 of the Study

\begin{tabular}{llll}
\hline & $\begin{array}{l}\text { Pre-SSC } \\
\text { Mean } \pm \text { SD }\end{array}$ & $\begin{array}{l}\text { SSC } \\
\text { Mean } \pm \text { SD }\end{array}$ & $\begin{array}{l}\text { Post-SSC } \\
\text { Mean } \pm \text { SD }\end{array}$ \\
\hline Day one & $23.07 \pm 6.5$ & $29.04 \pm 10.2$ & $28.64 \pm 10.3$ \\
Day five & $22.00 \pm 4.73$ & $28.92 \pm 8.23$ & $26.46 \pm 6.51$ \\
\hline
\end{tabular}

\section{TEWL}

TEWL demonstrated a similar pattern of increase from preSSC to SSC and then decrease from SSC to post-SSC on Days 1 and 5 (Table 5 and Fig. 3, 4). A 3 X 3 X 2 Repeated Measures Mixed Models Design was used to analyze of TEWL across time. Specifically, the model tested comparisons in TEWL made across ASSESSMENTS, PERIODS, and DAYS, as well as all possible interactions. No significant main effect differences of TEWL in ASSESSMENT data collection points and DAYS were identified. However, significant differences in TEWL were found across PERIODS $(F(2,18)=17.49 ; p<0.001)$. It should be noted that all mean scores are based on Least Squares Mean estimates. In examining the differences in TEWL across PERIODS, TEWL scores during the Pre-SSC period $($ LSMeans $=22.54)$ were significantly lower than TEWL scores during the SSC period $($ LSMeans $=28.98)$ (Tukey-Kramer adjusted $\mathrm{p}<$ $0.001)$. TEWL scores during Pre-SSC (LSMeans $=22.54$ ) were significantly lower than TEWL scores during Post-SSC $($ LSMeans $=27.55)($ Tukey-Kramer adjusted $p<0.001)$. No significant differences were found comparing TEWL scores during SSC and TEWL scores during Post-SSC. None of the four interactions was significant.

\section{Infection}

Only one infant developed a hospital-acquired infection during hospitalization and that infection was clinically evident 7 days after SSC ended. None of the mothers reported any signs or symptoms of infection during the first thirty days post-discharge.

\section{Discussion}

The study investigated the effects of 5 consecutive days of 90 minutes SSC sessions on stratum corneum hydration and transepidermal water loss by recording and comparing values on Days 1 and 5 while infants were in an incubator (30 minutes pretest), in SSC (90 minutes test), and back in an in-

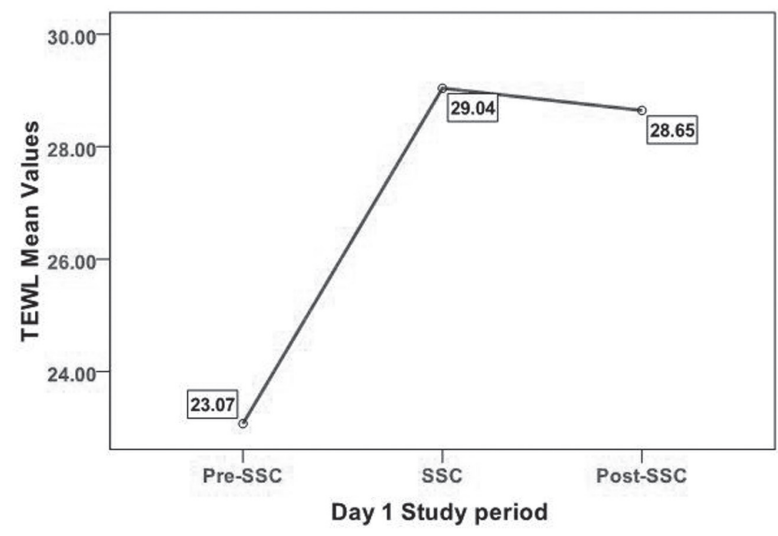

Figure 3. TEWL on Day 1 across all periods.

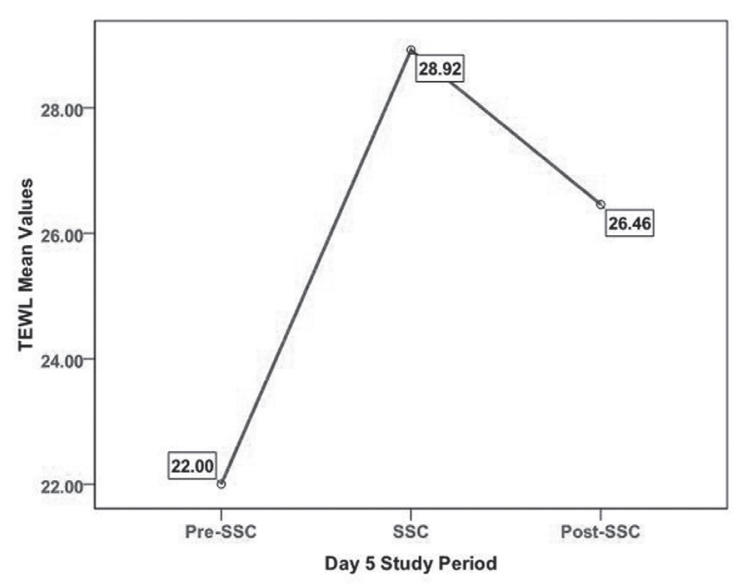

Figure 4. TEWL on Day 5 across all periods.

cubator (30 minute posttest). The number of infections during and after hospitalization were tracked and showed that only one infant had a positive blood culture seven days after SSC stopped and no infants had signs of infection within 4 weeks of discharge. Reviewing the risk factors for developing infection revealed that the infant was at higher risk of infection because he was on CPAP and had an intravenous catheter longer than other infants; these findings are consistent with Cohen-Wolkowiez and colleagues [34]. Because SSC's positive effect on reducing number of infections has been found to be limited to the time when SSC is being given or only shortly thereafter [35], repeated sessions of SSC need to be conducted to determine prevention of infection.

Stratum corneum hydration and transepidermal water loss are indications of skin barrier function in preterm infants. Stratum corneum hydration, while controlling for transepidermal water loss, increased from pre-SSC to SSC and decreased in post-SSC on Days 1 and 5 of the study. Thus, SSC contributed to better stratum corneum hydration and did 
so probably because skin-to-skin contact provides a degree of occlusivity at the skin surface, thereby also causing skin wetness. Skin wetness contributes to higher transepidermal water loss readings, which were present during SSC. The increased hydration finding is similar to the effects of skin occlusion in diapered areas in infants during the first 2 - 3 weeks of life but not at 4 weeks of life due to skin maturation $[36,37]$. Transepidermal water loss is known to be increased in infant skin areas in which skin-to-skin contact occurs, such as in the cubital fossa when flexion of the forearm occurs due to newborn postures [38].

Additionally, residual effects of SSC were seen. Skin hydration was higher after SSC than before SSC on both days, and skin hydration was higher before SSC on Day 5 than it was on Day 1, suggesting that the increases in skin hydration were not just momentary, but lasting. Such a possibility is congruent with the physiology of the skin in that skin hydration changes are commonly detectable for several days [39, 40]. Residual effects of SSC have been found for 1 - 2 hours after SSC terminates [41], so these results contribute new information related to length of residual effects of SSC. Residual effects on TEWL also were suggested by data showing that TEWL after SSC was higher than TEWL before SSC, possibly due to SSC or maturation of the infant's skin over the five days of study [42].

The increase in stratum corneum hydration during SSC is an important finding. A hydrated stratum corneum maintains flexibility and is able to strengthen the cornified cell envelopes, increasing the strength of the corneocytes responsible for the strength of the stratum corneum [43]. Good moisturization (hydration) of the skin allows the stratum corneum to perform fully and optimizes the ability of the body to resist pathogens. Enhanced hydration also optimizes strength and flexibility of the skin, preventing an influx of unwanted chemicals and inflammatory responses [44]. Good stratum corneum hydration is the most basic need for adequate skin barrier function.

Two ways to hydrate the skin exist. One way is to conserve the skin's natural moisture by using an ointment; the other way is to add moisture. In SSC mother's chest, temperatures increase and sweating may occur [26, 45, 46], introducing maternally produced hydration into the skin-to-skin interface. Thus, SSC may work as an occlusive moisturizing agent that adds moisture and has an immediate hydration effect on the stratum corneum, as mentioned before. Yet, the amount of SSC producing any given amount of additional water at the skin surface is not known and needs to be investigated because excess hydration or prolonged hydration may predispose the skin to inflammation and irritation [47]. Fortunately, the stratum corneum usually gains only minimal moisture from the addition of water to the skin surface [44], suggesting that the 2-hour sessions of SSC may not be a problem, but the effects of repetitive SSC and/or 24/7 SSC on skin integrity need to be evaluated because prolonged oc- clusion may also affect skin microbial flora, hydration and TEWL [48].

The levels of transepidermal water loss recorded during the incubator periods were expected because TEWL remains high in preterm infants for several weeks after birth [49]. The increases in TEWL during SSC were probably due to the mother adding to the moisture available at the SSC interface that was available for evaporation rather than the infant's skin bringing more water to the surface that was available for evaporation. Accordingly, water came to the infant's outer layers of skin, raising the possibility that SSC may act as a mechanical humectants (an agent, usually chemical, that attracts the water from inner skin layers to the outer layer of the skin [50]), and further studies should be conducted to quantify maternal contributions to the high TEWL values. Additionally, Wesley and Malbach explained that, in addition to finding difference between black and white skin, TEWL of both races increases with skin temperature increase [51]. In the current study, infant skin temperatures increased during the two hours of SSC and the increase was similar to that previously noted in many other studies [52]. Because high TEWL implies concomitant loss of heat [23], one might think the thermal management of the preterm neonate during SSC could be compromised; however, body heat loss does not occur during SSC $[52,53]$ and basal metabolic rate does not rise when the infant's temperature rises [54] because mothers conduct heat to the infant during SSC [25, $26,46]$, rather than the infant increasing heat production by his/her own metabolism.

The number of infection results need to be considered cautiously because of the small sample size and studies with larger sample size are warranted to determine the effects of SSC on infection rate. Nonetheless, SSC may help reduce the number of infections in other ways than modifying skin hydration. For example, SSC reduces infant stress [55, 56]. Stress affects the epidermis and the stratum corneum in ways that disrupt skin barrier functioning [57] by increasing corticosteroid secretion which reduces the production of lipids and intercellular lamellae in the stratum corneum, reducing skin barrier strength and water content of the skin $[44,58]$. Endogenous glucocorticoids increase permeability of the skin barrier, contributing to stress-induced infections [59]. Thus, the multiple changes occurring in the skin of the stressed infant places the infant at risk for skin barrier dysfunction and infants in intensive care are usually stressed and have higher than normal levels of circulating cortisol $[55,60$, 61]. SSC may minimize skin barrier dysfunction by ameliorating stress $[22,55,56]$.

A limitation of the study was that statistical control for ambient humidity was not possible due to the small number of ambient humidity data points (ambient humidity was taken at the beginning of each period, not at each assessment). Because humidity influences transepidermal water loss, future studies should include more frequent measure- 
ment of ambient humidity. Future studies should also include equal numbers of dark- and light-pigmented skin subjects as well as measuring skin barrier function at different skin areas (i.e. the infant's back) during each period to control for site difference and occlusion effects. Also, future studies could examine maternal skin hydration and transepidermal water loss simultaneous to the infant's and test for any correlations between maternal and infant's values to determine presence of physiologic synchrony as a mechanism for change. Especially because physiologic synchrony between mothers and infants during SSC has been found in many biomarkers [26, $45,46,62-65]$.

\section{Conclusions}

Hospital acquired infection is an important problem in preterm infants [1]. The data reported here are encouraging and suggest that SSC may be an occlusive agent to promote skin barrier functioning and assist the infant's underdeveloped immunoprotective functions in minimizing the possibility of hospital-acquired infections. The study has begun fulfilling the recommendation that SSC be tested as a strategy to improve skin hydration [4], but reveals that evaporative loss may be higher during SSC than during incubator care, and that the higher transepidermal evaporated water loss values may not necessarily be detrimental because few infections occurred even in its presence. Until definitive data are obtained through randomized controlled trials, clinical application of SSC to improve barrier functions and be recognized as a mechanism contributing to reduced infections is premature.

\section{Acknowledgement}

The research was funded in part by the Foundation of Neonatal Nursing Research and Education to the first author and by a RIG Grant from the Bolton School of Nursing to the second author.

\section{References}

1. Mortality and Acute Complications in Preterm Infants. In Preterm Birth: Causes, Consequences, and Prevention, Behrman RE, Butler AS, eds. (Washington, DC. National Academies Press), 2007; pp. 313-345.

2. Darmstadt GL, Saha SK, Ahmed AS, Ahmed S, Chowdhury MA, Law PA, Rosenberg RE, et al. Effect of skin barrier therapy on neonatal mortality rates in preterm infants in Bangladesh: a randomized, controlled, clinical trial. Pediatrics 2008;121(3):522-529.

3. Yoshio H, Tollin M, Gudmundsson GH, Lagercrantz H, Jornvall H, Marchini G, Agerberth B. Antimicrobial polypeptides of human vernix caseosa and amniotic fluid: implications for newborn innate defense. Pediatr Res 2003;53(2):211-216.

4. Chiou YB, Blume-Peytavi U. Stratum corneum maturation. A review of neonatal skin function. Skin Pharmacol Physiol 2004;17(2):57-66.

5. Thaver D, Zaidi AK. Burden of neonatal infections in developing countries: a review of evidence from community-based studies. Pediatr Infect Dis J 2009;28(1 Suppl):S3-9.

6. Aly NY, Salmeen HN, Lila RA, Nagaraja PA. Pantoea agglomerans bloodstream infection in preterm neonates. Med Princ Pract 2008;17(6):500-503.

7. Barros FC, Victora CG, Barros AJ, Santos IS, Albernaz E, Matijasevich A, Domingues MR, et al. The challenge of reducing neonatal mortality in middle-income countries: findings from three Brazilian birth cohorts in 1982, 1993, and 2004. Lancet 2005;365(9462):847-854.

8. Carey AJ, Saiman L, Polin RA. Hospital-acquired infections in the NICU: epidemiology for the new millennium. Clin Perinatol 2008;35(1):223-249, x.

9. Orsi GB, d'Ettorre G, Panero A, Chiarini F, Vullo V, Venditti M. Hospital-acquired infection surveillance in a neonatal intensive care unit. Am J Infect Control 2009;37(3):201-203.

10. Yasmin S, Osrin D, Paul E, Costello A. Neonatal mortality of low-birth-weight infants in Bangladesh. Bull World Health Organ 2001;79(7):608-614.

11. Stoll BJ, Hansen N, Fanaroff AA, Wright LL, Carlo WA, Ehrenkranz RA, Lemons JA, et al. Late-onset sepsis in very low birth weight neonates: the experience of the NICHD Neonatal Research Network. Pediatrics 2002;110(2 Pt 1):285-291.

12. Mamula KB. Medicare policy change forces hospitals to step up monitoring. Pittsburgh Business Times, available online at: http://www.bizjournals.com/pittsburgh/ stories/2008/04/14/story4.html April 11, 2008.

13. Pear R. Medicare Says It Won't Cover Hospital Errors New York Times. www.mha.org. April 11, 2007.

14. Bahl R, Martines J, Ali N, Bhan MK, Carlo W, Chan KY, Darmstadt GL, et al. Research priorities to reduce global mortality from newborn infections by 2015. Pediatr Infect Dis J 2009;28(1 Suppl):S43-48.

15. Ohlsson A, Lacy JB. Intravenous immunoglobulin for preventing infection in preterm and/or lowbirth-weight infants. Cochrane Database Syst Rev 2004;(1):CD000361.

16. Martines J, Paul VK, Bhutta ZA, Koblinsky M, Soucat A, Walker N, Bahl R, et al. Neonatal survival: a call for action. Lancet 2005;365(9465):1189-1197.

17. Larson EL, Cimiotti J, Haas J, Parides M, Nesin M, Della-Latta P, Saiman L. Effect of antiseptic handwashing vs alcohol sanitizer on health care-associated infections in neonatal intensive care units. Arch Pediatr Adolesc 
Med 2005;159(4):377-383.

18. Larson E, Silberger M, Jakob K, Whittier S, Lai L, Della Latta P, Saiman L. Assessment of alternative hand hygiene regimens to improve skin health among neonatal intensive care unit nurses. Heart Lung 2000;29(2):136142.

19. Darmstadt GL, Batra M, Zaidi AK. Oral antibiotics in the management of serious neonatal bacterial infections in developing country communities. Pediatr Infect Dis J 2009;28(1 Suppl):S31-36.

20. Darmstadt GL, Batra M, Zaidi AK. Parenteral antibiotics for the treatment of serious neonatal bacterial infections in developing country settings. Pediatr Infect Dis J 2009;28(1 Suppl):S37-42.

21. Charpak N, Ruiz-Pelaez JG, Figueroa de CZ, Charpak Y. Kangaroo mother versus traditional care for newborn infants $</=2000$ grams: a randomized, controlled trial. Pediatrics 1997;100(4):682-688.

22. Charpak N, Ruiz JG, Zupan J, Cattaneo A, Figueroa Z, Tessier R, Cristo M, et al. Kangaroo Mother Care: 25 years after. Acta Paediatr 2005;94(5):514-522.

23. Agren J, Sjors G, Sedin G. Ambient humidity influences the rate of skin barrier maturation in extremely preterm infants. J Pediatr 2006;148(5):613-617.

24. Fransson AL, Karlsson H, Nilsson K. Temperature variation in newborn babies: importance of physical contact with the mother. Arch Dis Child Fetal Neonatal Ed 2005;90(6):F500-504.

25. Karlsson H. Skin to skin care:heat balance. Arch Dis Child Fetal Neonatal Ed 1996;75(2):F130-132.

26. Ludington-Hoe SM, Nguyen N, Swinth JY, Satyshur RD. Kangaroo care compared to incubators in maintaining body warmth in preterm infants. Biol Res Nurs 2000;2(1):60-73.

27. Ibe OE, Austin T, Sullivan K, Fabanwo O, Disu E, Costello AM. A comparison of kangaroo mother care and conventional incubator care for thermal regulation of infants $<2000 \mathrm{~g}$ in Nigeria using continuous ambulatory temperature monitoring. Ann Trop Paediatr 2004;24(3):245-251.

28. Modi N. Management of fluid balance in the very immature neonate. Arch Dis Child Fetal Neonatal Ed 2004;89(2):F108-111.

29. Ludington-Hoe SM, Anderson GC, Simpson S, Hollingsead A, Argote LA, Rey H. Birth-related fatigue in 34-36-week preterm neonates: rapid recovery with very early kangaroo (skin-to-skin) care. J Obstet Gynecol Neonatal Nurs 1999;28(1):94-103.

30. Hammarlund K, Sedin G. Transepidermal water loss in newborn infants. III. Relation to gestational age. Acta Paediatr Scand 1979;68(6):795-801.

31. Asembergiene J, Gurskis V, Kevalas R, Valinteliene R. Nosocomial infections in the pediatric intensive care units in Lithuania. Medicina (Kaunas) 2009;45(1):29-
36.

32. Okah FA, Wickett RR, Pickens WL, Hoath SB. Surface electrical capacitance as a noninvasive bedside measure of epidermal barrier maturation in the newborn infant. Pediatrics 1995;96(4 Pt 1):688-692.

33. Courage and Khazaka Electronic GmbH. (2007) Available at: http://www.courage-khazaka.de.

34. Cohen-Wolkowiez M, Benjamin DK, Jr., Capparelli E. Immunotherapy in neonatal sepsis: advances in treatment and prophylaxis. Curr Opin Pediatr 2009;21(2):177-181.

35. Cerezo MR, de Leon R, Gonzales BJ. Mother-child early contact with "the mother kangaroo" program and natural breast feeding. Review Latino American Perinatology 1992;(12):54-60.

36. Visscher MO, Chatterjee R, Ebel JP, LaRuffa AA, Hoath SB. Biomedical assessment and instrumental evaluation of healthy infant skin. Pediatr Dermatol 2002;19(6):473481.

37. Visscher MO, Chatterjee R, Munson KA, Pickens WL, Hoath SB. Changes in diapered and nondiapered infant skin over the first month of life. Pediatr Dermatol 2000;17(1):45-51.

38. Yosipovitch G, Maayan-Metzger A, Merlob P, Sirota L. Skin barrier properties in different body areas in neonates. Pediatrics 2000;106(1 Pt 1):105-108.

39. Hoeger PH, Enzmann CC. Skin physiology of the neonate and young infant: a prospective study of functional skin parameters during early infancy. Pediatr Dermatol 2002;19(3):256-262.

40. Tabata N, O'Goshi K, Zhen YX, Kligman AM, Tagami $\mathrm{H}$. Biophysical assessment of persistent effects of moisturizers after their daily applications: evaluation of corneotherapy. Dermatology 2000;200(4):308-313.

41. Cleary GM, Spinner SS, Gibson E, Greenspan JS. Skinto-skin parental contact with fragile preterm infants. J Am Osteopath Assoc 1997;97(8):457-460.

42. Visscher MO, Maganti S, Munson KA, Bare DE, Hoath SC. Early adaptation of human skin following birth; a biophysical assessment. Skin Research Technology 1999;5:213-220.

43. Harding CR, Long S, Richardson J, Rogers J, Zhang Z, Bush A, Rawlings AV. The cornified cell envelope: an important marker of stratum corneum maturation in healthy and dry skin. Int J Cosmet Sci 2003;25(4):157167.

44. Fore-Pfliger J. The epidermal skin barrier: implications for the wound care practitioner, part II. Adv Skin Wound Care 2004;17(9):480-488.

45. Bergstrom A, Okong P, Ransjo-Arvidson AB. Immediate maternal thermal response to skin-to-skin care of newborn. Acta Paediatr 2007;96(5):655-658.

46. Bystrova K, Matthiesen AS, Vorontsov I, Widstrom AM, Ransjo-Arvidson AB, Uvnas-Moberg K. Maternal axillar and breast temperature after giving birth: effects of 
delivery ward practices and relation to infant temperature. Birth 2007;34(4):291-300.

47. Rustemeyer T, Frosch PJ. Occupational skin diseases in dental laboratory technicians. (I). Clinical picture and causative factors. Contact Dermatitis 1996;34(2):125133.

48. Aly R, Shirley C, Cunico B, Maibach HI. Effect of prolonged occlusion on the microbial flora, $\mathrm{pH}$, carbon dioxide and transepidermal water loss on human skin. J Invest Dermatol 1978;71(6):378-381.

49. Agren J, Sjors G, Sedin G. Transepidermal water loss in infants born at 24 and 25 weeks of gestation. Acta Paediatr 1998;87(11):1185-1190.

50. Man MM, Feingold KR, Thornfeldt CR, Elias PM. Optimization of physiological lipid mixtures for barrier repair. J Invest Dermatol 1996;106(5):1096-1101.

51. Wesley NO, Maibach HI. Racial (ethnic) differences in skin properties: the objective data. Am J Clin Dermatol 2003;4(12):843-860.

52. Ludington-Hoe SM, Morgan K, Abouelfettoh A. A clinical guideline for implementation of SSC with premature infants 30 or more weeks postmenstrual age. Advances in Neonatal Care 2008;8(3S):S3-S23.

53. Moore ER, Anderson GC, Bergman N. Early skin-toskin contact for mothers and their healthy newborn infants. Cochrane Database Syst Rev 2007;(3):CD003519.

54. Bauer J, Sontheimer D, Fischer C, Linderkamp O. Metabolic rate and energy balance in very low birth weight infants during kangaroo holding by their mothers and fathers. J Pediatr 1996;129(4):608-611.

55. Morelius E, Theodorsson E, Nelson N. Salivary cortisol and mood and pain profiles during skin-to-skin care for an unselected group of mothers and infants in neonatal intensive care. Pediatrics 2005;116(5):1105-1113.

56. Anderson GC, Chiu SH, Dombrowski MA, Swinth JY, Albert JM, Wada N. Mother-newborn contact in a randomized trial of kangaroo (skin-to-skin) care. J Obstet Gynecol Neonatal Nurs 2003;32(5):604-611.
57. Denda M, Tsuchiya T, Elias PM, Feingold KR. Stress alters cutaneous permeability barrier homeostasis. Am J Physiol Regul Integr Comp Physiol 2000;278(2):R367372.

58. Mooncey S, Giannakoulopoulos X, Glover V, Acolet D, Modi N. The effect of mother-infant skin-to-skin contact on plasma cortisol and Beta-endorphin concentrations in preterm infants. Infant Behavior and Development 1997;20(4):553-557.

59. Choi EH, Brown BE, Crumrine D, Chang S, Man MQ, Elias PM, Feingold KR. Mechanisms by which psychologic stress alters cutaneous permeability barrier homeostasis and stratum corneum integrity. J Invest Dermatol 2005;124(3):587-595.

60. Gitau R, Modi N, Gianakoulopoulos X, Bond C, Glover V, Stevenson J. Acute effects of maternal skin-toskin contact and massage on saliva cortisol in preterm babies. Journal of Reproductive Infant Psychology 2002;20(2):83-88.

61. D'Apolito KC. State of the science: procedural pain management in the neonate. J Perinat Neonatal Nurs 2006;20(1):56-61.

62. Feldman R. Maternal-infant contact and child development: Insights from the kangaroo Intervention. In Low Cost Approaches to Promote Physical and Mental Health. Theory, Research, and Practice, L'Abate L, ed. (Springer Publishers, New York), 2007; pp. 323-351.

63. Feldman R, Eidelman AI. Skin-to-skin contact (Kangaroo Care) accelerates autonomic and neurobehavioural maturation in preterm infants. Dev Med Child Neurol 2003;45(4):274-281.

64. Ludington-Hoe SM, Lewis T, Morgan K, Cong X, Anderson L, Reese S. Breast and infant temperatures with twins during shared Kangaroo Care. J Obstet Gynecol Neonatal Nurs 2006;35(2):223-231.

65. Neu M, Laudenslager ML, Robinson J. Coregulation in salivary cortisol during maternal holding of premature infants. Biol Res Nurs 2009;10(3):226-240. 